\title{
DISCOVERING RESUME INFORMATION USING LINKED DATA
}

\author{
Ujjal Marjit $^{1}$, Kumar Sharma $^{2}$ and Utpal Biswas ${ }^{3}$ \\ ${ }^{1}$ C.I.R.M, University Kalyani, Kalyani (West Bengal) India \\ sic@klyuniv.ac.in \\ ${ }^{2}$ Department of Computer Sc. \& Engg, University Kalyani, Kalyani (West Bengal) India \\ kumar.asomegmail.com \\ ${ }^{3}$ Department of Computer Sc. \& Engg, University Kalyani, Kalyani (West Bengal) India \\ utpal01indyahoo.com
}

\begin{abstract}
In spite of having different web applications to create and collect resumes, these web applications suffer mainly from a common standard data model, data sharing, and data reusing. Though, different web applications provide same quality of resume information, but internally there are many differences in terms of data structure and storage which makes computer difficult to process and analyse the information from different sources. The concept of Linked Data has enabled the web to share data among different data sources and to discover any kind of information while resolving the issues like heterogeneity, interoperability, and data reusing between different data sources and allowing machine process-able data on the web.

In this paper we present Linked Data approach to discover resume information enabling the task aggregation, sharing and reusing the information among different resume information providers and organizations. We also demonstrate experimental results on semantically rich resume information.
\end{abstract}

\section{KEYWORDS}

Semantic Web, Linked Data, Resume, Provenance

\section{INTRODUCTION}

In the present day, the Web 2.0 mainly suffers from data sharing and reusing between two data sources. The Web 2.0 is basically a web of documents which is well formatted and presented inside the HTML documents but the semantic of data is missing due to which computer is unaware of what data or information is being presented inside the HTML documents. In Web 2.0, data is hardly process-able by the computer because data are presented and decorated inside the HTML documents and hence the computer is unaware of these hidden data. It just blindly displays the information inside documents without knowing the meaning of displayed information. Nevertheless, these challenges are overtaken by the Semantic Web technology where data is part of the web and the web directly talks with data to data instead of documents as in the Web 2.0. Hence, the Semantic Web is also known as a Web of Data. According to Sir Tim Berners Lee, Semantic Web is "an extension of the current Web in which information is given well-defined meaning, better enabling computers and people to work in cooperation." Semantic Web or Web 3.0 has changed the way we present information into the web. Semantic Web uses graph database to store its data and allows data to be self-described in a more structured way so 
that the computer can easily process and analyse the data. RDF (Resource Description Framework) and ontology are the main building blocks of Semantic Web. RDF [1] is a language for representing information about resources in the web. RDF describes resource's properties and relationship whereas ontology is a part of data modelling which provides a collection of terms and concept to share information. RDF also acts as a unifying data model for information and resources in the Semantic Web. In RDF, each resource has its properties and relationships in a triple form- Subject, Predicate, and Object. Subject represents a resource that is being described, Predicate represents resource's properties or relationship, and the Object represents property value, which may be literal as well as another resource. Each of these three triples, except for literal values, can be described using URIs (Uniform Resource Identifier) that uniquely identifies a resource on the web. Hence, the Semantic Web is built on top of RDF, which uses URIs to identify data. Once the information is in RDF format then it will be easy for computer to process, search, analyse and discover information on the web.

Resume is a document managed by a person containing his/her personal information as well as information about academic and work experiences, which is used when applying for a job. Resume is also used by Expert Finders to find experts during a recruitment process in an organization. A resume produces detail information about a person's job, career, Skills used, information about organization he/she previously worked etc., so that Expert Finders can easily select those experts depending on their requirements. At present, several web applications have been emerged where people can edit, create and update their resumes and keep there for further reference so that organizations can easily find it through web applications. However, present web or Web 2.0 does not define semantics that are reliable. For instance, creation process of resumes in traditional web is done by editing and writing in some documents and then some of these data are stored in file system or database. This would mainly affect the task of information sharing and data reusing. In such cases, the computer is unaware of the presented information into the web documents.

In this paper, we discuss the task of efficient discovery and use of resume information using Linked Data technology. The paper is divided as follows: we discuss the concept of Linked Data in Section 2. Section 3 presents the related work. We present our proposed work in section 4, section 5 presents experimental results, and Section 6 concludes our work.

\section{LINKED DATA}

In 2006, Tim Berners Lee first coined a new concept, a further extension to the existing Semantic Web called Linked Data to make the resources linked up with other resources. Linked Data is an approach on Semantic Web, which enables data publishers to publish and interlink the structured data on the Web of Data as well as data consumers to discover and integrate data from different data sources. Since the data in existing web are not linked to each other, the Linked Data allows each data to be linked and shared the information and knowledge between them by using RDF and URIs enabling the task of data sharing and data reusing. Sir Tim Berners-Lee [2] [3] have proposed four principles for publishing the structured data on the web as following:

1. Use URIs for naming things.

2. Use HTTP URIs, so that people can look up those names.

3. When someone looks up a URI, provide useful information, using the standards (RDF, SPARQL).

4. Include links to other URIs, so that they can discover more things.

The use of URIs for naming things helps uniquely identify the resources such as people, places, or any real-world entities. Since the HTTP protocol is the universal access mechanism, so using 
International Journal of Web \& Semantic Technology (IJWesT) Vol.3, No.2, April 2012

HTTP URIs makes the resource discoverable and any one can look up the resource by dereferencing the HTTP URI. The Linked Data principles also suggest the use of a common data model, i.e., RDF. To share data in the Web of Data it is important to agree on a common data model which facilitates several applications to be easily queried and consumed the data. Because of its unifying and single data model where all entities in the world are uniquely identified, the task of data sharing would become easy. Since anyone can publish any kind of data on the Web of Data, the publishers from around the world such as many individuals, organizations, and business domains has started to publish their data of any kind such as governmental data, geographical data, information about books, media data, etc. making the Web of Data a global graph of data as shown in the Figure 1.

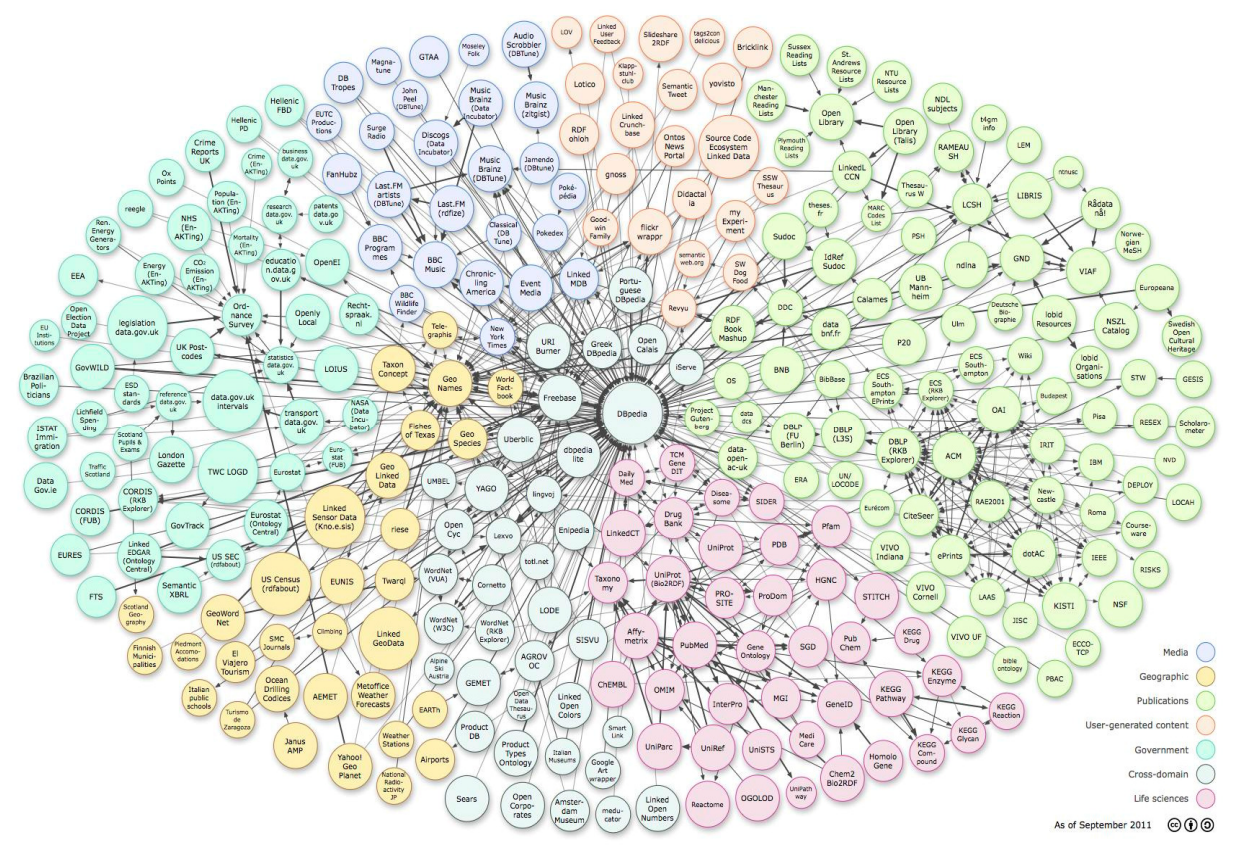

Figure 1: Linked Open Data Cloud, a global data graph (Adapted from [4])

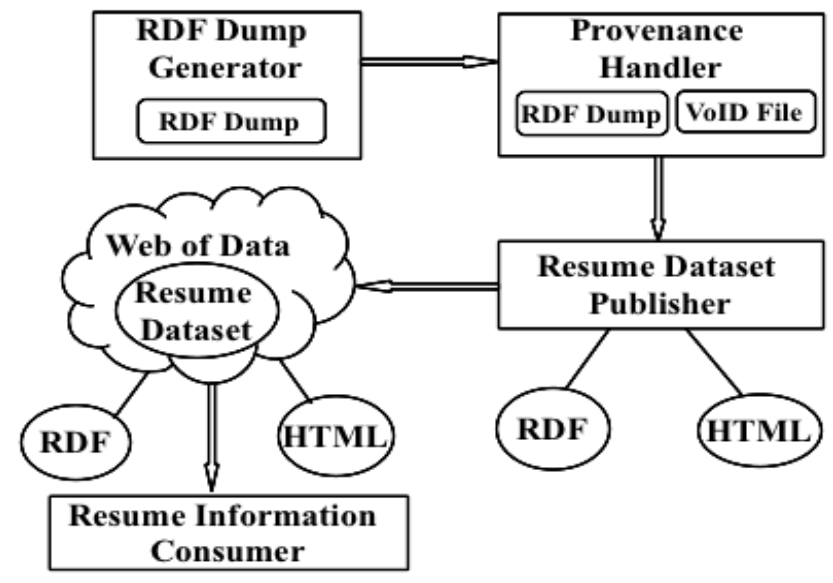

Figure 2: Work flow of publishing and consuming resume information into the Web of Data 
International Journal of Web \& Semantic Technology (IJWesT) Vol.3, No.2, April 2012

\section{RELATED WORK}

Uldis Bojars et. al. [5] has introduced ResumeRDF ontology to model resume using RDF data model. There is also an extension to FOAF [6] to support description of resume such as foaf:publication property to describe information about publications. ResumeRDF has rich set of classes and properties to describe resume information. ResumeRDF [7] expresses resume information using two namespaces:-

- http://captsolo.net/semweb/resume/0.2/cv.rdf - Resume ontology

- http://captsolo.net/semweb/resume/0.2/base.rdf - Property value taxonomy

Using ResumeRDF a rich set of resume information can be described such as a person's detail information, his/her reference information, skill information, work and education experiences etc. We will be relying on ResumeRDF as well as FOAF [8] ontology to present our experimental data. Authors also discussed about discovery and aggregation of resume information on the web using existing social networking and online community sites. However, using social networking and community sites, web still suffers from data sharing and reusing. Also we argue that data integration from multiple sources will remain an issue. People can export resumes from their personal or shared accounts and referenced candidates. Also the applications can not rely on such sites to consume and display the resume information. As we discussed Linked Data connects distributed data sources across the web and also allows SPARQL query service to access the resources. We believe that using Linked Data to discover and aggregate resume information will bring high benefits to the people and organizations.

\section{USING LiNKED DATA TO DISCOVER RESUME INFORMATION}

Linked Data is a great way for publishing and connecting data from different sources such as distributed organizations, individuals, governments etc. Our key objective is to publish, discover and aggregate the semantically rich information contained in resume of a person. As we have discussed about Semantic Web that enhances data by inserting semantics so that machine can easily process it. Figure 2 shows our work flow regarding publishing the resume RDF dataset into the Web of Data, where the RDF Dump Generator generates resume information represented in RDF. Once the Provenance Handler receives RDF Dump it will generate the provenance metadata of the Resume RDF dump, i.e., the Provenance Handler is responsible for VoID file generation which tells about Resume RDF dataset description, licensing information, vocabularies used, and other provenance metadata. The Resume Dataset Publisher is responsible for generating various representation of the resume data such as RDF for machine purpose and HTML representation for human user which is used during content negotiation. As soon as the RDF dump is published on the Web of Data by the Resume Dataset Publisher, it is available on the Web and is ready for the consumers to consume the resume information. Similarly, the VoID file representing provenance metadata of the resume dataset can be published in the same way as the RDF dataset is published, either separately or in the same storage. Resume Information Consumer asks for resume information from the Web of Data where the consumer can request for either the representation of data such as RDF and HTML or any other representation.

Having data both for machine as well as human or semantically and syntactically strong then information in the web becomes easily discoverable and reusable. Linked Data allows resources to be openly published and connected thereby discovering more information in the system. The main benefit using Linked Data approach is that every piece of information will contain richness of description while having interlinking and interoperability between different data sources. 
The Semantic Web follows graph database to store any kind of data. The Semantic Web consists of resources related to other resources or literal values. One important benefit using graph database for storing data is that we can always break any sort of data and re-combine or merge them with other similar data sources to discover new sort of relationships. In our case, if a resume information of a person is already described by one company and if another company or an organization wants to describe the resume information for the same person, then the later can directly refer to the former's data source or can merge with itself instead of creating the new one, i.e., Linked Data also reduces redundancy by uniquely describing resources and sharing across the Linked Open Data. Thus, any resource in resume information should not be described or defined again if it is already exists. In that case we can refer to the existing resource by using owl:sameAs/rdf:seeAlso properties.

Linked Data allows anyone to contribute the resources that can be reused and recombine with other resources in the system. Therefore, the resume resources can always be extensible with the resources of web friendly identifiers.

If we publish our resume dataset into Web of Data using Linked Data then it would help in many areas ranging from sharing and reusing data, and it also reduces the task of Expert Finders by directly relying on RDF data. In addition, every resource such as a company, person, Skill inside a resume will have their unique HTTP URI and is available through worldwide by dereferencing these URIs. Efficient SPARQL queries over resume dataset would help Expert Finders to easily find the experts with a very less effort. These queries will help to reduce the job of reading document based resumes and also using standard ontologies will bring unification in the system. For this, we need to build applications to explore resume information by consuming information from the Web of Data.

\subsection{Consuming Resume Resources}

After publishing the resume information resources into the web it becomes a part of the Linked Open Data, to be consumed by Linked Data applications in order to make them useful. The Web of Data allows applications to consume the data very easily due to the open nature of the web. Since Linked Data uses a standard or common data model to represent its data, we do not have to rely on different data representation formats as in Web 2.0. Tom Heath et. al. [2] has described that the Linked Data architecture is open and enables the discovery of new data sources at run time so that the Linked Data applications can get the use of new data as soon as it is available in the Web of Data. So, in order to consume the resources available in the resume dataset it is mandatory to have Linked Data applications to display or visualize the required information. Presently there are two categorization of Linked Data applications, as described in [2], as Generic Applications and Domain-specific Applications. Generic Applications such as Linked Data browsers and Linked Data search engines can browse and crawl the data by following RDF links and display the information integrating from various sources, for example Disco-Hyperdata [9] is a Linked Data browser that displays information while navigating the Semantic Web. Domainspecific applications are those that display the information by consuming Linked Data based on the user's requirement. One can build effective application by deciding the user requirement and what resume information should populate to know detail about a resume.

Applications can also rely on Linked Data APIs. Though, Linked Data allows publishing information using a standard format, the underlying RDF data model and access to the RDF resources using SPARQL technology are not yet familiar to the Web 2.0 users. RESTFul APIs over RDF triple stores provide easy access to the RDF data. Linked Data APIs may return resources in simple XML or JSON formats generated from the RDF triple representation. Garrote et. al. [10] presents possible ways to build Linked Data APIs which focuses on transforming 
International Journal of Web \& Semantic Technology (IJWesT) Vol.3, No.2, April 2012

SPARQL queries into SQL queries over relational database and transforming HTTP requests to SPARQL queries. This way application should not directly depend on the technologies like RDF and SPARQL while relying on RESTful Linked Data APIs.

\subsection{Design Requirements}

Christian Bizer et. al. [11] have presented detail picture about how to publish Linked Data on the Web, the Linked Data architecture, how to choose URIs, which vocabularies to use to represent information, setting RDF links to other data sources etc. While describing resources in the resume dataset and interlinking them into Web of Data using Linked Data approach some design requirements should be considered. These requirements are for both resume information and the Linked Data which are as follows:

\subsubsection{Resume Format}

Type of resume depends on the publishers. A resume may contain Academic or Business information or both. Business resume reveals a person's detail about job experience, whereas Academic resume focuses on providing education background information such as degree type, publications etc. Publisher should also focus on resume structure which depends on the compilation of the resume which may follow reverse chronological order and functional structures.

\subsubsection{Use of Taxonomy and URI scheme}

There should be new taxonomies and URI assignment schemes, as discussed in [6], for real-world objects or resources described in resume dataset in order to easily dereferencable. Use of plain literals for certain property values may lead to ambiguity. The real world objects can be Skill name identification, persons, organizations educational institutions, etc.

\subsubsection{Public or Private}

Since the nature of Linked Data is open in nature especially when we are using Linked Open Data (LOD) project, but there can be dataset of type both public and private. It all depends on the publishers whether or not to make data open for all. If the resume dataset should not be publicly accessible then the publishers make them private while publishing data with an explicit license [12].

\subsubsection{Use of confidential information}

While describing certain information such as personal or references information one has to be careful regarding their personal information, applicable laws and regulations [5]. There may be some information that should not be openly described, these considerations should be taken during system designing.

\subsubsection{Provenance Information}

Since any one can publish any kind of data into the Web of Data, Data could suffer from trust and quality. In order to introduce quality data and to provide trust on resume resources, publishers should also publish the Provenance metadata of their dataset. Provenance information helps users or data consumers to determine the quality and trustworthiness of the data. Provenance metadata can also be published in the same way as we publish Linked Data. VoID [13] is a vocabulary for describing RDF datasets and also it provides guidelines for deploying and discovering VoID 
descriptions of a dataset. VoID tells about how and under what conditions to access the data as well as provenance and licensing information. Keith et al. [14] describes everything about VoID and has presented some use cases how VoID can be applied to describe RDF datasets.

\section{EXPERIMENTS}

In this section we present our experiment on RDF based resume information where a dataset containing description of resume information is generated and then SPARQL queries are used to find the needed information. We have used terms from ResumeRDF ontology to describe the resume information and FOAF ontology to describe the information about persons. Figure 3 shows a short picture of resume RDF graph, which describes a resume of a person named KS and the work history named WorkHistory\#KS having its job title, job type and starting date description. Similarly, it shows other information such as skill, company, title, whether or not the resume is active, and the date of last update.

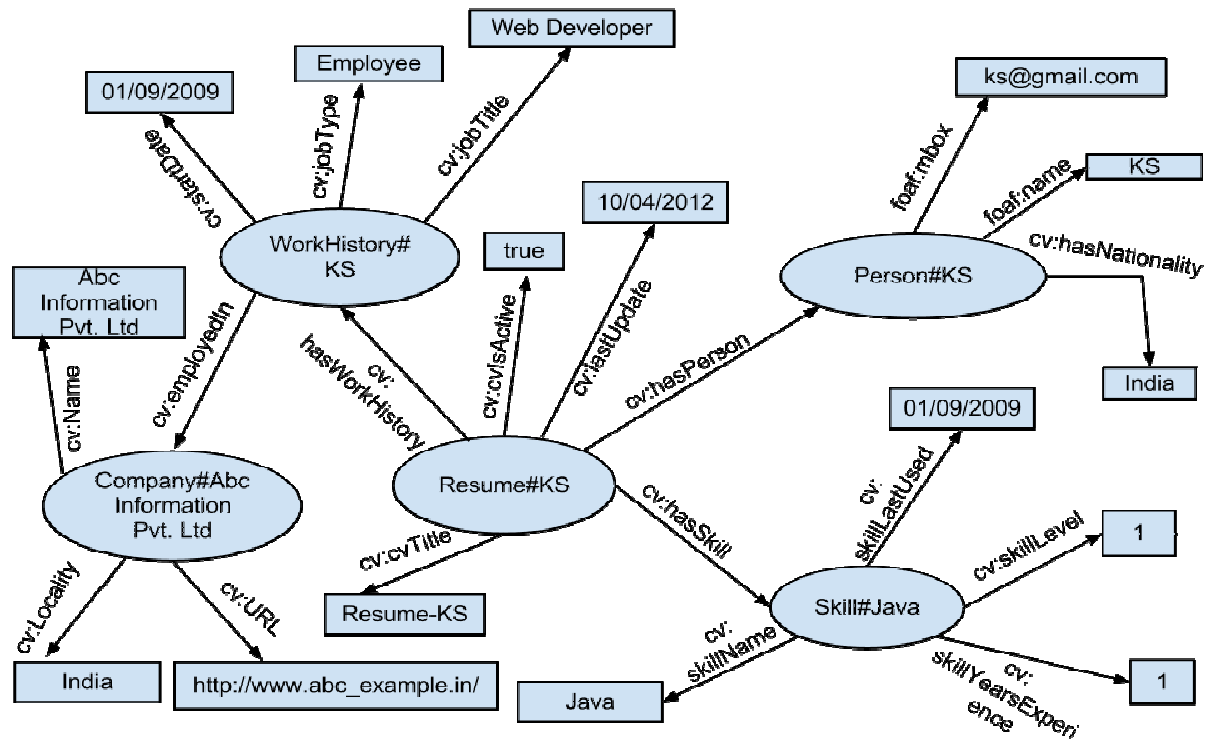

Figure 3: Resume RDF Graph

The RDF data is generated using Jena 2.6.4 and then it is stored using TDB as dataset. This dataset is assumed as a part of the Web of Data and is used for our experimental purpose. A resume dataset as such contains information about both academic and business resumes along with the information about companies and Educational organizations. For experimental purpose, we have used following set of resume information:-

\subsection{Personal Information}

Personal information contains information about a person, such as name, nickname, address, email-id, etc. It also includes information about referenced persons. The property cv:about person is used to indicate person whose resume is being described. Normally, personal information is described using FOAF and Vcard however ResumeRDF has introduced new properties 
cv:birthPlace, cv:hasNationality, cv:hasCitizenship, cv:hasDrivingLicense, etc. which are missing in FOAF, to describe personal information related to resume. Table-1 shows a code snipped of RDF description about person.

Table 1: Description of person using cv:aboutPerson

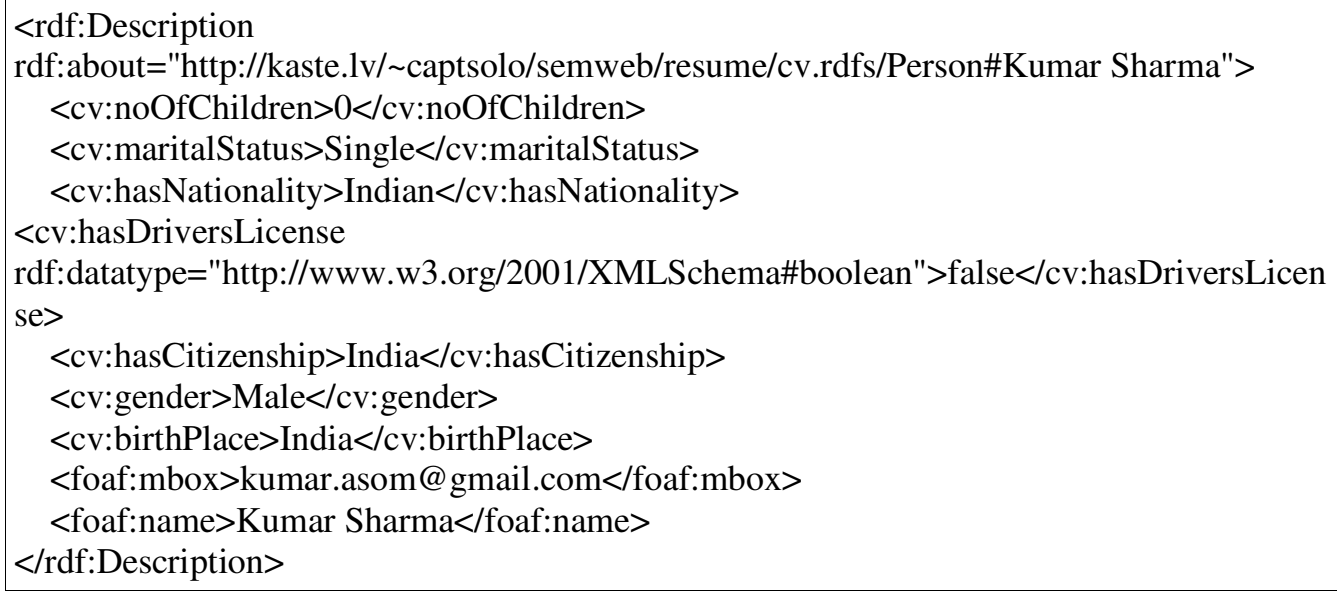

\subsection{Organizational Information}

Organization information can be divided into two sections- Work Experience and Academic Experience. Work Experience provides the information about job such as the name of the current company, starting date, total years of experience etc. The property cv:hasWorkHistory is used to describe work history. Similarly, Academic Experience tells about the information about person's educational experience which is described by the properties cv:hasEducation, cv:degreeType, cv:eduDescription, cv:studiedIn, etc. Table-2 shows RDF description code snipped describing the work history of a person.

Table 2: Describing work experience using cv:hasWorkHistory

<rdf:Description
rdf:about="http://kaste.lv/ captsolo/semweb/resume/cv.rdfs/WorkHistory\#kumar">
<cv:isCurrent
rdf:datatype="http://www.w3.org/2001/XMLSchema\#boolean">true</cv:isCurrent>
<cv:jobType>Employee</cv:jobType>
<cv:jobDescription>Software development</cv:jobDescription>
<cv:jobTitle>Web Developer</cv:jobTitle>
<cv:endDate rdf:datatype="http://www.w3.org/2001/XMLSchema\#date">-</cv:endDate>
<cv:startDate rdf:datatype="http://www.w3.org/2001/XMLSchema\#date">01/09/2009
</cv:startDate>
<cv:employedIn
rdf:resource="http://kaste.lv/ captsolo/semweb/resume/cv.rdfs/Company\#Abc Information
Pvt. Ltd"/>
</rdf:Description>




\subsection{Skill Information}

Skill is the main topic in a resume information system which describes a person's skill information such as skill name, years of experience in a particular skill, skill level etc. cv:skillName, cv:hasSkill, cv:skillLastUsed, cv:skillLevel(0-5), cv:skillYearsExperience, properties are used to describe skill related information as shown in Table-3.

Table 3: Description of skill using cv:hasSkill

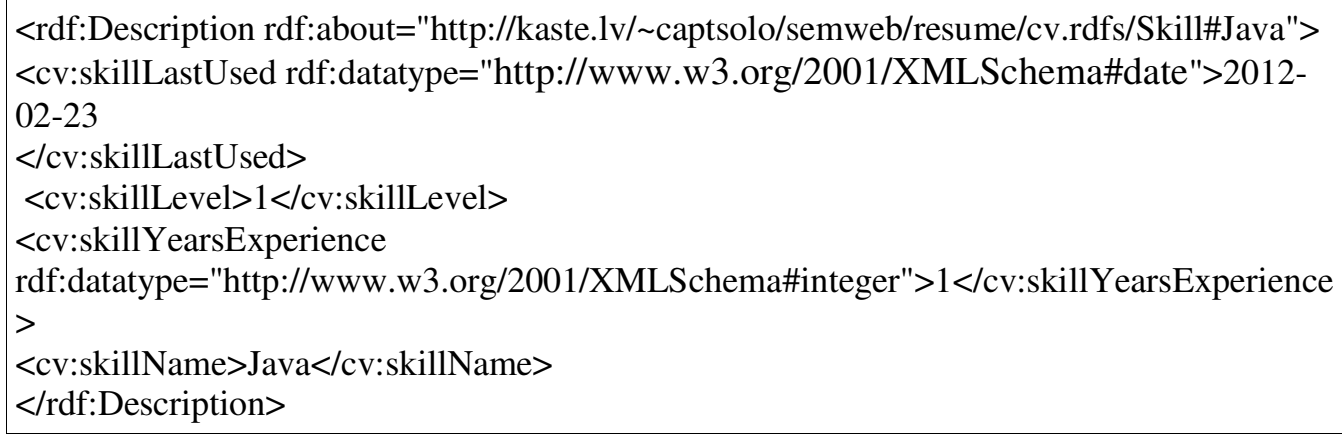

\subsection{Other information}

Apart from the above information ResumeRDF has certain properties such as cv:cvTitle, cv:cvDescription to describe resume's special information, whether or not the current resume is active using cv:cvIsActive property, date of update using cv:lastUpdate etc. Table-4 shows a detail RDF description about resume.

Table 4: Description about a resume

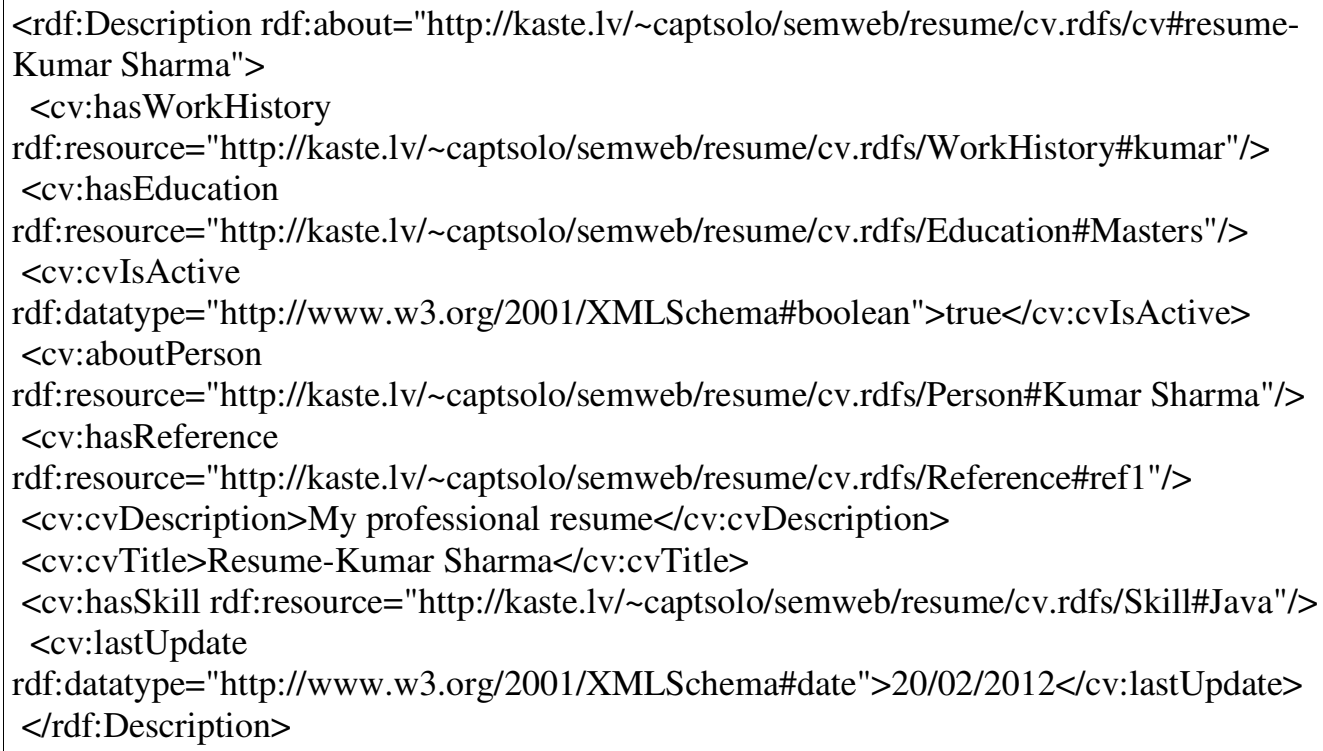

As we have the resume information semantically described using RDF we can now use this information in various areas. The information is always machine processable, we can explore this information through the web or applications relying on the RDF dataset. We can also use it to 
find the required information while executing SPARQL queries. Once the dataset is ready anywhere in the world, one can build effective applications relying on the Linked Data and SPARQL queries to explore the information. Such application would really help Expert Finders to find the required information easily. For example the query "Give me some resume who works in Java and have more than 5 years of experience" the SPARQL query would look like as shown in Table-5.

Table 5: Querying resume information whose skill is Java \& 5 years experience

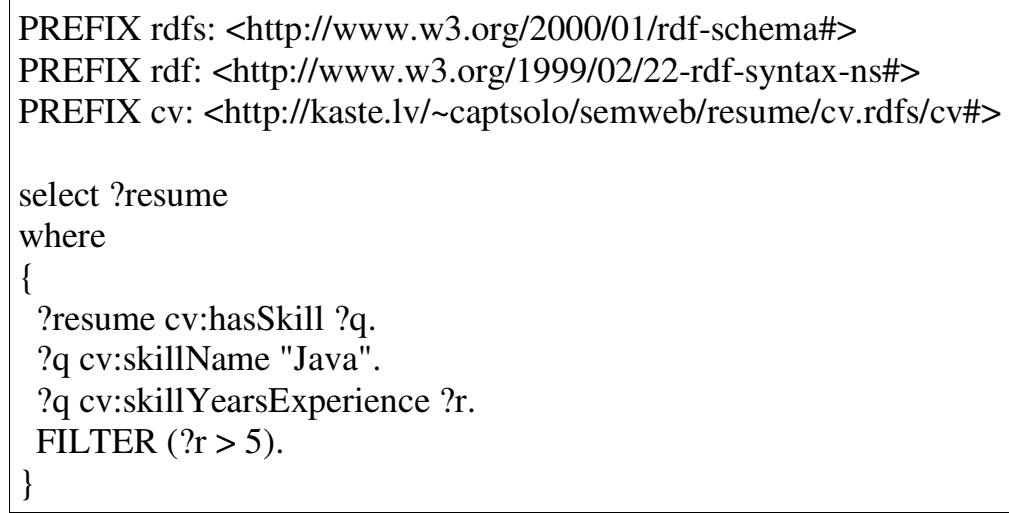

Similarly, the query "Select resumes and display person's email-id whose skill is Java." would look like as in Table-6.

Table 6: Querying personal information whose skill is Java \& 5 years experience

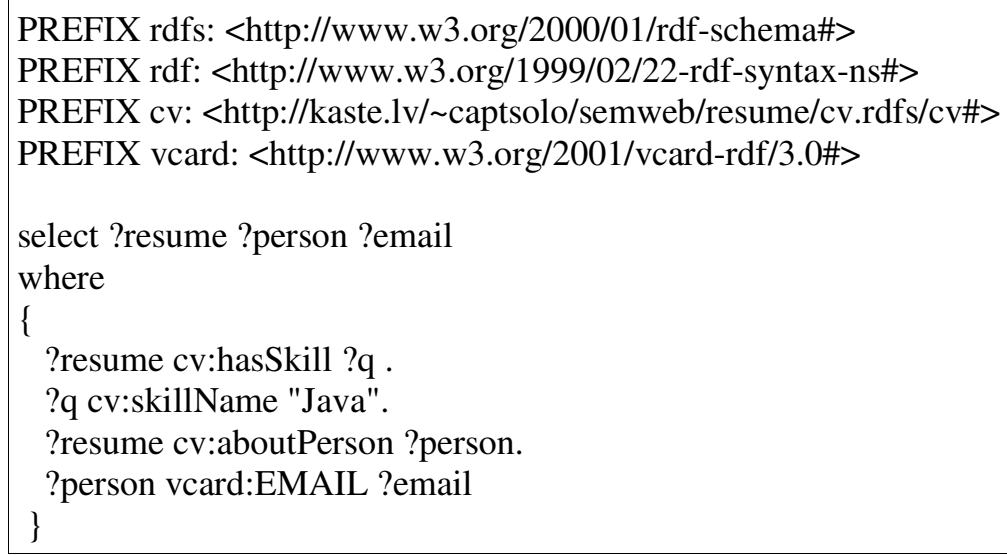

\section{CONCLuSion}

In this paper, we have presented Linked Data approach to discover and aggregate resume information into the Web of Data. We believe that using Linked Data technology will not only ease the task of discovery and aggregation but also resolves heterogeneity, interoperability, and data reusing between multiple data sources, and allows every piece of resume information to be self described as well as discoverable using HTTP URIs. We also presented the benefits using Linked Data and to consume the resume information from the Web of Data using Linked Data applications. A sample snippet of resume informations have also been presented in order to better understanding of resume RDF datasets. Our future work will include investigation of Linked Data 
International Journal of Web \& Semantic Technology (IJWesT) Vol.3, No.2, April 2012

Applications to consume the resume resources from the Web of Data in order to analyse the performance and usability. We also plan to publish the provenance metadata to make trusted and quality data.

\section{REFERENCES}

[1] RDF Primer, available at http://www.w3.org/TR/rdf-primer/

[2] Tom Heath, Christian Bizer, "Linked Data Evolving the Web into a Global Data Space". Synthesis Lectures on the Semantic Web: Theory and Technology, Morgan \& Claypool Publishers, ISBN $978160845431,2011$.

[3] Tim Berners-Lee, T. Linked Data. 2006.

http://www.w3.org/DesignIssues/LinkedData.html

[4] Richard Cyganiak and Anja Jentzsch at http://lod-cloud.net/

[5] Uldis Bojars, John G. Breslin, "ResumeRDF: Expressing Skill Information on the Semantic Web". The 1st International ExpertFinder Workshop (EFW 2007), Berlin, Germany, 16 January 2007.

[6] Uldis Bojars, “Extending FOAF with Resume Information”. Available at http://www.w3.org/2001/sw/Europe/events/foafgalway/papers/pp/extending_foaf_with_resume/

[7] ResumeRDF, available at http://rdfs.org/resume-rdf/

[8] FOAF ontology, avialable at http://xmlns.com/foaf/spec/

[9] Disco-Hyperdata Linked Data browser, available at http://www4.wiwiss.fu-berlin.de/rdf_browser/

[10] Antonio Garrote, María N. Moreno García, "RESTful writable APIs for the web of Linked Data using relational storage solutions", Next Generation Web Services Practices (NWeSP), 2011 7th International Conference, 19-21 Oct. 2011.

[11] Christian Bizer, Tom Heath, Richard Cyganiak. "How to Publish Linked Data on the Web". Available at http://www4.wiwiss.fu-berlin.de/bizer/pub/LinkedDataTutorial/

[12] The Linking Open Data Cloud Diagram, http://richard.cyganiak.de/2007/10/lod/

[13] Describing Linked Datasets with the VoIDVocabulary, W3C Interest Group Note 03 March 2011, http://www.w3.org/TR/void/

[14] Keith Alexander, Richard Cyganiak, Michael Hausenblas, Jun Zhao. "Describing Linked Datasets, On the Design and Usage of voiD, the Vocabulary Of Interlinked Datasets”. LDOW2009, 2009. 
International Journal of Web \& Semantic Technology (IJWesT) Vol.3, No.2, April 2012

\section{Authors}

Ujjal Marjit is the System-in-Charge at the C.I.R.M.(Centre for Information Resource Management), University of Kalyani. He obtained his M.C.A. degree from Jadavpur University, India in 2000. Currently he is pursuing his Ph.D. from University of Kalyani, India. His vast areas of research interest reside in Web Service, Semantic Web, Semantic Web Service, Ontology, Knowledge Management, e-Governance as well as Software Agents etc. More than twenty nine of his papers have been published in the several reputed national and international conferences and journals.

Kumar Sharma is a Research Scholar of the Department of Computer Science \& Engineering, University of Kalyani, India. He obtained his Bachelor degree (B.C.A) from University of North Bengal, India in 2006, and M.C.A. degree from University of Kalyani, India in 2009. His research interests include Semantic Web, Linked Data and Web technology.

Dr. Utpal Biswas received his B.E, M.E and $\mathrm{PhD}$ degrees in Computer Science and Engineering from Jadavpur University, India in 1993, 2001 and 2008 respectively. He served as a faculty member in NIT, Durgapur, India in the department of Computer Science and Engineering from 1994 to 2001. Currently, he is working as an Associate Professor in the department of Computer Science and Engineering, University of Kalyani, West Bengal, India. He is a co-author of about 58 research articles in different journals, book chapters and conferences. His research interests include Optical Communications, Ad-hoc and Mobile Communications, Sensor Networks, Semantic Web Services, E-governance etc. 